\title{
Near-Field Optical Microscopy of Defects in Cholesteric Oligomeric Liquid Crystal Films
}

\author{
Svetlana G. Lukishova \\ The Institute of Optics, University of Rochester, Rochester, \\ New York, USA
}

\author{
Ansgar W. Schmid \\ Laboratory for Laser Energetics, University of Rochester, Rochester, \\ New York, USA
}

This paper describes formation of 2-D-hexagonal structures with a periodicity $\sim 0.5-0.8 \mu \mathrm{m}$ in the defects of thin films of cholesteric oligomeric liquid crystals prepared by the evaporation of the solvent from the oligomer solution on the substrate. These regular arrays were observed by scanning near-field optical and concurrent atomic force microscopy. The mechanisms considered are both Bénard-Marangoni and buoyancy convections induced by solvent evaporation and air-bubble creation around the condensed water droplets from the air during evaporative cooling. Hexagonal structures prepared by this method can be used in photonic devices for emission enhancement, for instance, in liquid crystal lasers and single photon sources with oligomeric liquid crystal hosts.

Keywords: cholesteric oligomeric films; hexagonal arrays; near-field microscopy

The authors acknowledge the support by the U.S. Army Research Office under Award No. DAAD19-02-1-0285 and National Science Foundation Award ECS-0420888. The work was also supported by the U.S. Department of Energy Office of Inertial Confinement Fusion under Cooperative Agreement No. DE-FC03-92SF19460, the University of Rochester, and the New York State Energy Research and Development Authority. The support of DOE does not constitute an endorsement by DOE of the views expressed in this article.

Receipt of CLC oligomer from F. Kreuzer of Wacker, Munich is gratefully acknowledged. The authors thank K. Marshall and J. Starowitz for advice and support in the optical material laboratory, O. Lavrentovich and S. Shiyanovskii for discussion and providing useful references.

Address correspondence to Svetlana G. Lukishova, The Institute of Optics, University of Rochester, Rochester, NY 14627, USA. E-mail: sluk@lle.rochester.edu 


\section{INTRODUCTION}

Fabrication of highly ordered, light-wavelength-scale structures for light manipulation and control is one of the primary tasks of nanoscience applications in photonics. Planar-aligned cholesteric liquid crystals (CLCs) widely used in display technology and laser photonics are well-known for their 1-D photonic bandgaps. Here we report on 2-D-self-assembly into hexagonal patterns by thin-film defects of Wacker-cyclic-tetrasiloxane CLC oligomer [1]. This selfassembly is recorded with subwavelength resolution simultaneously by a near-field optical and an atomic-force microscope (AFM). Created in this manner, 2-D photonic crystal material doped with appropriate dye can be used for fluorescence control in photonic devices, for instance, oligomeric LC lasers [2], and efficient single photon sources for quantum information and communication, using oligomeric LC hosts [3-5].

The structure of this paper is as follows. Section 2 describes oligomeric CLC film preparation. Conventional optical polarizing microscopy (wide-field) images of defects in these films are presented. Section 3 describes the experimental results on the scanning near-field optical microscopy and the AFM imaging of the defects in Wacker oligomeric CLCs showing the hexagonally ordered arrays. Possible mechanisms of the self-assembly are discussed in Section 4. Section 5 concludes the paper.

\section{PREPARATION OF OLIGOMERIC CLC FILMS}

For our experiments, we used liquid crystal cyclic-tetrasiloxane LC-4627 with maximum selective reflection wavelength $\lambda_{\mathrm{o}}=2.2 \mu \mathrm{m}$. It was received from Consortium für Elektrochemische Industrie, Munich, Germany, a subsidiary of Wacker Chemie. The oligomer powder was dissolved either in chlorobenzene or in methylene chloride with approximately $8.4-15.6 \%$ concentration of Wacker oligomer by weight. Lower concentration of oligomer with lower solution viscosity did not provide film self-assembly reported in present paper. We prepared oligomer thin films on clean Corning cover glass slips $\left(25 \mathrm{~mm} \times 25 \mathrm{~mm}, 170 \mu \mathrm{m}\right.$ thickness) by holding the slip at close to $90^{\circ}$ angle and dripping the oligomer solution on to it. The solvent evaporated very quickly under the air flow of a fume hood.

Figure 1 shows representative optical-polarizing-microscope images of three samples prepared from solutions with different concentrations of the oligomer. $4 \times-10 \times$-magnification images (top set) reveal the appearance of domain structure where each cell is surrounded 


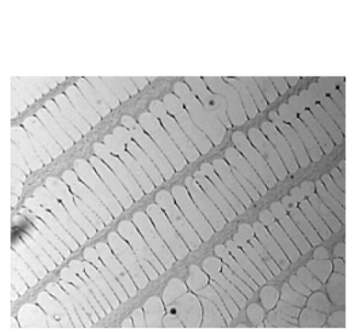

$4 X$

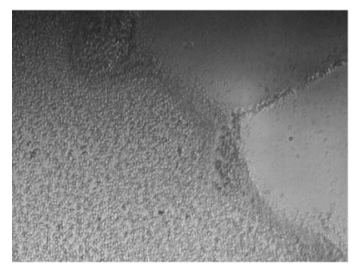

$40 x$

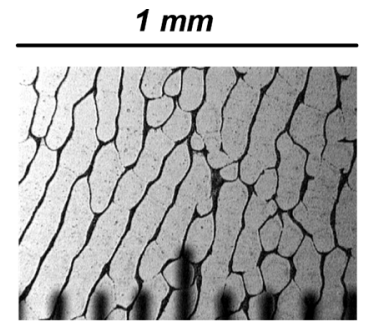

$10 x$

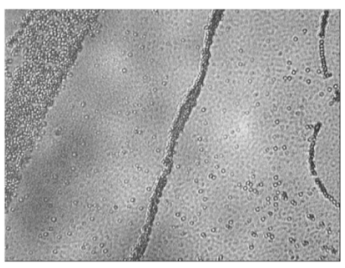

$40 x$

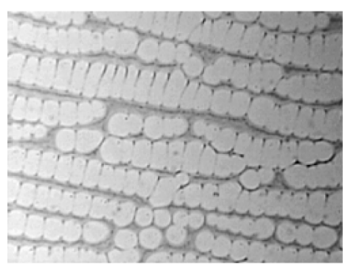

$4 X$

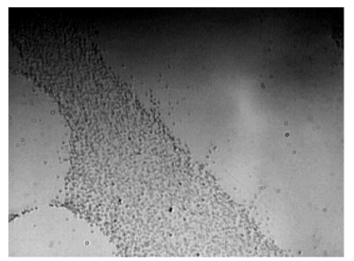

$40 x$

FIGURE 1 Representative selection of conventional polarizing-opticalmicroscopy images from three different samples of Wacker CLC oligomer films on glass substrates showing the cell domains separated by the defects (top set of images with $4 \times$ and $10 \times$ magnifications of the objective) and the defect areas (bottom set of images) with $40 \times$ magnification.

by boundaries. The widths of the domains are approximately $50-100 \mu \mathrm{m}$. Their shapes vary from circles and ovals to long stripes with near 1-mm-lengths. Similar cell domains have been observed in Ref. [6] in the process of evaporation of solvent in which both nematic and polymer were dissolved.

The bottom set of images in Figure 1 shows the film defects with $40 \times$ magnification of the objective. Even conventional optical microscopy reveals a regular, self-organized patterning.

To define the thickness of the film, an AFM of a razor-blade-cut of the sample of Figure 1, top-center was made. This film was prepared from a solution with the lowest oligomer concentration $(\sim 8.4 \%$ weight concentration) and showed a thickness of $65 \mathrm{~nm}$ in an area free of defects.

\section{NEAR-FIELD OPTICAL MICROSCOPY AND AFM OF THE DEFECTS IN FILMS OF WACKER CLC OLIGOMERS}

A cantilever SNOM (WiTec alpha-SNOM) in "tapping" mode with $\sim 100$-nm tip aperture enables both near-field optical microscopy and 
Optical near-field images AFM-images

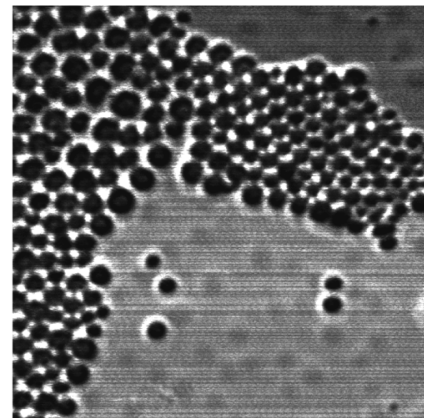

$15 \mu \mathrm{m} \times 15 \mu \mathrm{m}$

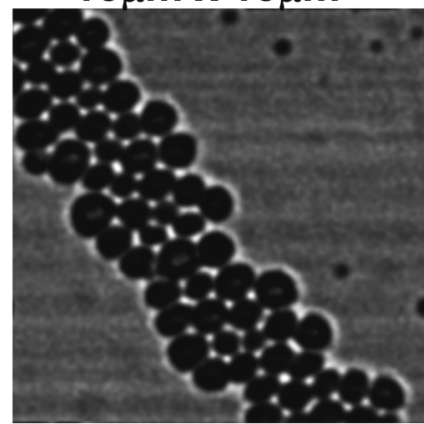

$10 \mu \mathrm{m} \times 10 \mu \mathrm{m}$

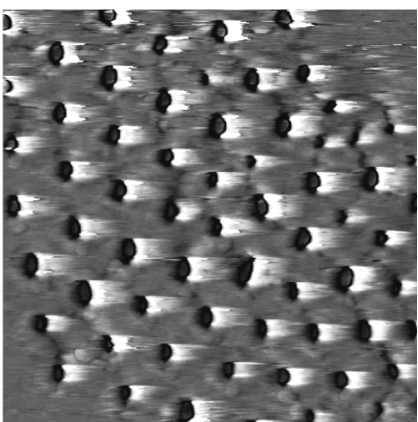

$5 \mu \mathrm{m} \times 5 \mu \mathrm{m}$

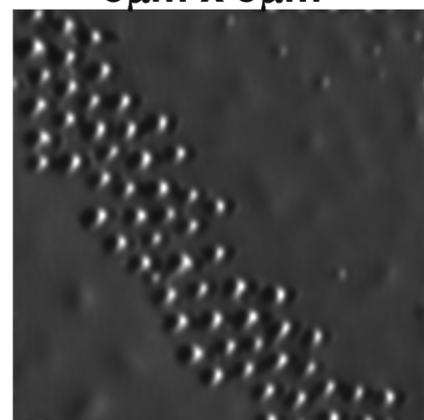

$10 \mu \mathrm{m} \times 10 \mu \mathrm{m}$

FIGURE 2 Near-field optical microscopy (left) and AFM (right) images of Wacker oligomer film defects with hexagonally ordered arrays. Bottom images were taken simultaneously.

simultaneous topographic imaging [7]. All images were taken of the sample of Figure 1, top-center.

Figure 2, left shows the scanning near-field optical microscopy images (left) and AFM images (right) of hexagonal ordering in defects of the oligomer layer. The bottom images (both nearfield microscopy and AFM) were taken simultaneously during the scan.

Another set of images of a different area with hexagonal ordering (Fig. 3) represents the AFM image (left) and near-field images of this area for two different polarizations.

Figure 4 shows one more set of the near-field images: the whole defect with $50 \mu \mathrm{m} \times 50 \mu \mathrm{m}$ scanning area - left, and its parts with $10 \mu \mathrm{m} \times 10 \mu \mathrm{m}$ (center) and $5 \mu \mathrm{m} \times 5 \mu \mathrm{m}$ (right) area scans. 


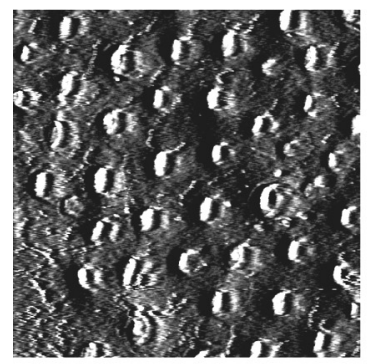

$5 \mu \mathrm{m} \times 5 \mu \mathrm{m}$

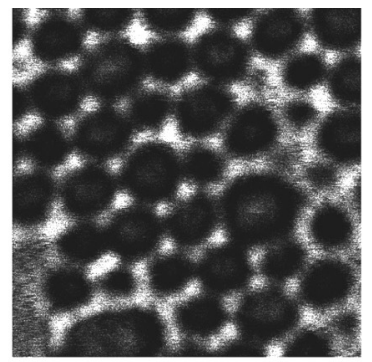

$5 \mu \mathrm{m} \times 5 \mu \mathrm{m}$

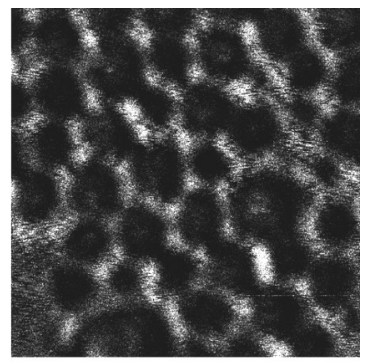

$5 \mu \mathrm{m} \times 5 \mu \mathrm{m}$

FIGURE 3 AFM (left) and near-field optical microscopy images (center and right) for two different polarizations of the laser beam showing a hexagonal ordering in Wacker oligomer film defects.

\section{MECHANISMS OF FORMATION OF THE CELLULAR DOMAINS AND REGULAR HEXAGONAL ARRAYS IN FILM DEFECTS}

In fluid mechanics, pattern formation during evaporation is a common phenomenon and the existence of cellular structures was recognized to be linked to surface tension and buoyancy, see, for instance, Ref. [8]. Convective movements originating from surface-tension gradients are known as Bénard-Marangoni convection. Both Bénard-Marangoni and buoyancy convections depend on the fact that solvent evaporation cools the liquid surface thereby increasing the surface tension as well as the density and leading to a stratification of the mixture $[6,8]$. Convection-induced regular patterning of PMMA and polystyrene has been obtained with the periodicity $\sim 3-10 \mu \mathrm{m}[9,10]$.

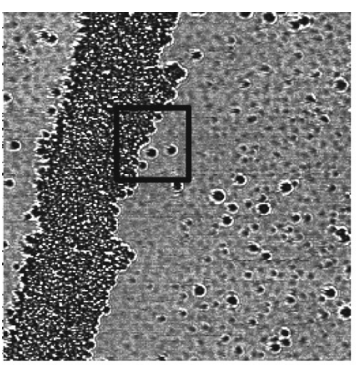

$50 \mu \mathrm{m} \times 50 \mu \mathrm{m}$

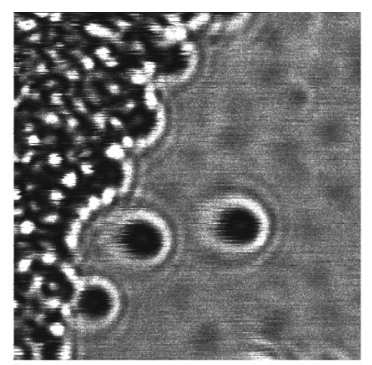

$10 \mu \mathrm{m} \times 10 \mu \mathrm{m}$

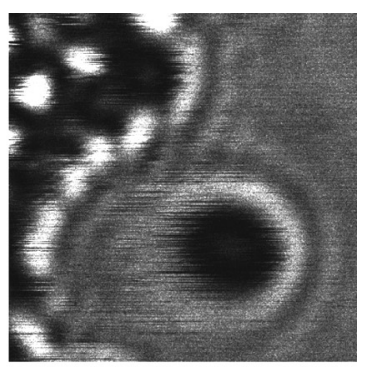

$5 \mu \mathrm{m} \times 5 \mu \mathrm{m}$

FIGURE 4 Near-field optical microscopy images of the same defect in the Wacker oligomer film with different scan areas. 
Regarding our experiments, we believe, that $\sim 50-100-\mu \mathrm{m}$ cellular domains may be the result of a convective motion. The movement of liquid solution along the close to the $90^{\circ}$ angle substrate may influence the shape of these convective-motion cells.

Although hexagonal array formation in the fluids have been reported for a pure evaporative convection [8], explaining such arrays in our experiments, we suggest another mechanism which has been detailed in Ref. [11]. Cooling by the evaporating solvent leads ambient moisture to condense on the hydrophobic mixture. Water droplets segregate and entrap into these self-assembling, hexagonally-ordered patterns [11,12], that, because of convection movement and flow movement by the liquid crystal medium along one direction, gets confined within cellular domains oriented parallel to the flow direction. Within minutes, the system returns to equilibrium with the water evaporated from the cavities leaving the air bubbles on the film surface (see, e.g., Fig. 4, center and right).

\section{CONCLUSION}

This paper presents the first results on 2-D hexagonal ordering in oligomeric CLC films during the solvent evaporation from the substrate in a specific range of oligomer concentrations. Both near-field optical microscopy and AFM showed the existence of such ordering with a periodicity of $\sim 0.5-0.8 \mu \mathrm{m}$. Combined action of convection and water droplet condensation during the evaporative cooling are the suggested mechanisms of the observed hexagonal patterns.

Our results are very promising for the fabrication of 2-D and 3-D phonic crystals for visible and near-IR spectral regions by this relatively simple method (see, for instance, Ref. [11]), but for obtaining the reproducible results with desired parameters, additional experiments need to be done.

\section{REFERENCES}

[1] Bunning, T. J. \& Kreuzer, F.-H. (1995). Trends in Polym. Sci., 3, 318.

[2] Shibaev, P. V., Kopp, V., Genack, A., \& Hanelt, E. (2003). Liquid Crystals, 39, 1391.

[3] Lukishova, S. G., Schmid, A. W., McNamara, A. J., Boyd, R. W., \& Stroud, C. R. (2003). Spec. issue on quantum internet technologies. IEEE J. Selected Topics in Quant. Electronics, 9(6), 1512.

[4] Lukishova, S. G., Schmid, A. W., Supranowitz, C. M., Lippa, N., McNamara, A. J., Boyd, R. W., \& Stroud, C. R. (2004). Special issue single photon: Detectors, applications and measurements methods. J. Mod. Optics, 51(9-10), 1535.

[5] Lukishova, S. G., Schmid, A. W., Knox, R. P., Freivald, P., McNamara, A. J., Boyd, R. W., Stroud, C. R., \& Marshall, K. L. (2006). Molec. Cryst. Liq. Crystal., 454, 403. 
[6] Golovataya, N. M., Kurik, M. V., \& Lavrentovich, O. D. (1990). Liquid Crystals, $7(2), 287$.

[7] www.witec-instruments.com.

[8] Mancini, H. \& Maza, D. (2004). Europhys. Lett., 66(6), 812.

[9] Mitov, Z. \& Kumacheva, E. (1998). Phys. Rev. Lett., 81, 3427.

[10] Kumacheva, E., Li, L., Winnik, M. A., Shinozaki, D. M., \& Cheng, P. C. (1997). Langmuir, 13, 2483.

[11] Srinivasarao, M., Collings, D., Philips, A., \& Patel, S. (2001). Science, 292, 79.

[12] Widawski, G., Rawiso, M., \& François, B. (1994). Nature, 369, 387. 